\title{
Process Modeling Semantics for Complex Business Environments
}

\author{
I.T. Hawryszkiewycz \\ School of Systems, Management and Leadership \\ University of Technology, Sydney \\ igorhait.uts.edu.au
}

\begin{abstract}
Process management is becoming more complex especially when business units work together to create new systems constructed from many components. The complexity arises both from the growing number of components and relationships as well as continual changes in product requirements and business arrangements. The complexity impacts on process management as support systems are needed to provide the communications and coordination to support the complex relationships and their continuing change. This paper proposes a systematic way to model such processes by developing the semantics to describe complex processes in meaningful ways. The semantics include perspectives other than those found in process flows to provide a more meaningful way to describe and model complex processes. The paper then outlines ways to convert the models to lightweight platforms that directly support the modeling concepts. The paper shows the application to complex tendering processes, which many of which now require greater flexibility and collaboration.
\end{abstract}

Keywords: Complexity, Modeling, Business Processes.

\section{Introduction}

Increasing complexity within the current business environment is introducing new approaches to system design. Such approaches must pay more attention to system complexity now found in the increasingly dynamic business environment. This complexity arises from an increasing trend to business networking and responding to changing service demands. One common example of such environments is supply chains based on business networking and usually supported by ERP systems. They appear in many industries as for example telecom [1] and automotive [2] industries. Complex tendering processes found in many government projects also include the coordination of different suppliers in large projects followed by the integration and testing of supplied components. Each supplier is often one component of the workflow and is required to provide a service that is coordinated by a project manager. Whereas ERP systems focus on optimizing information flows, the increasing complexity and greater emphasis on collaborative supply chains, requires other perspectives to be considered, in particular social networks and knowledge to continuously develop new knowledge to optimize and rearrange supply chain processes. Rye [3] for 
example calls for knowledge hubs to be established at all supply chain transitions. Pralahad and Krishnan [4] also argue that social networking will play an increasingly important role in such coordination. In many cases coordination is through the exchange of knowledge, much of it of a tacit nature, created by process participants.

These trends have a number of implications for the design of engineering systems and the management of processes. Such systems require support for collaboration between the different units to work towards common goals. These support systems must maintain awareness across the different processes, coordination within and between teams in the environment, and facilitate the knowledge sharing.

- The emergence of process ecosystems [5], where links between the different processes are continually changing and awareness must be maintained between process participants to keep track of outcomes in distant units that may impact on their own work,

- The trend to a more service oriented environment where systems must continually respond to changing customer needs requiring the continuous sharing of knowledge across units through the business processes, and

- Greater client involvement in the design [6] where solutions are created through collaboration between supplier network and the customer network.

The paper provides systematic ways to describe processes in such complex environments emphasizing the increasing role of social relationships [4] in knowledge creation. It particularly addresses the question as to whether new modelling concepts are needed to design such systems. The paper proposes that such new concepts can be derived from complexity theory. The paper identifies some such concepts and suggests that they become criteria in system design. It then defines how the criteria can be met using a number of perspectives to allow complexity to be managed in a systematic manner. It then describes modelling methods to describe systems from the different perspectives and the kinds of design processes needed to create systems to support complex processes.

\section{Design Guidelines from Complexity Theory}

To some people complexity is seen as arising from the interconnection on many objects. This is often referred to as combinatorial complexity. This can be the design of a complex communication systems or circuits as those found in modern day computer systems. Many of these can be solved by tools that deal with such complexity. Complex systems are seen to be different as they need to deal with unanticipated events that cannot be addressed using existing rules. Hence there is much more emphasis on social structures to address such problems and resolve them.

McElroy [7] identifies a number of fundamental ideas arising from complexity theory. These are illustrated in Figure 1, which identifies three main dimensions for design. These see the growing importance of knowledge management as the driver of innovation. Such knowledge must be developed as part of an increasingly complex environment that calls for increased emphasis on organizational learning. It stresses 


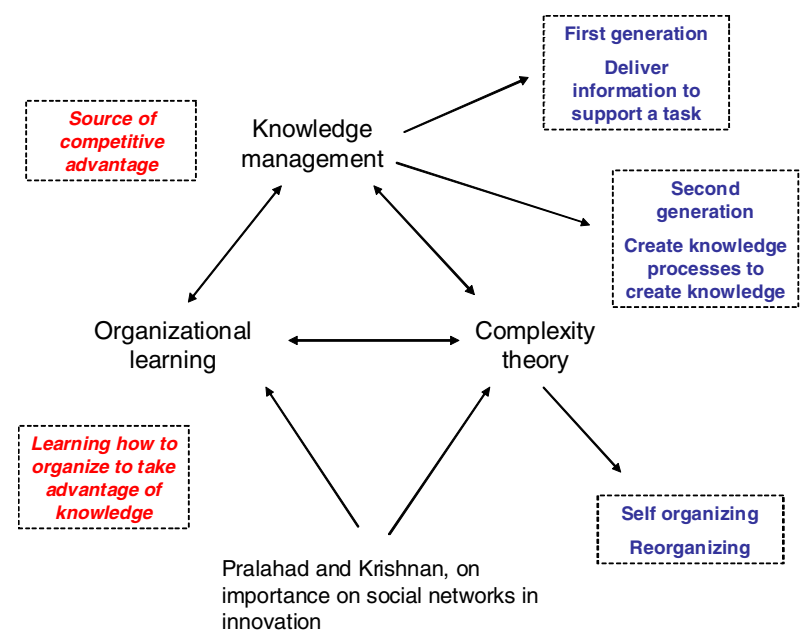

Fig. 1. Important Dimensions in Complexity Theory

that knowledge needs to be created during processes and not just information that may be consolidated to support a task. At the same time, Figure 1 also builds on the importance of social networks within complex systems by suggesting the appropriate networks e "injected" into systems to facilitate productive evolution.

In summary, the following design criteria are identified as important in system design. These are:

Learning both as organizations and individuals,

Knowledge capture and sharing,

Perception of the environment and responding to changes in the environment,

Communication and relationship building, and

Technology to provide system support.

These criteria are related as social structures must be chosen in ways that people collaborate to create new knowledge. At the same time they learn ways to do to things better and retain this knowledge for subsequent use and to support change. A number of papers such as that of Merali $[9,10]$ define the nature of change based on concepts of evolution found in complexity theory. These are derived from complexity theory and summarized [8] and in terms meaningful to system designers. These include:

- Ability to self-organize at local levels in response to wide variety of external changes

- Quick establishment of self-contained units that address well-defined parts of the environment

- Loose connections between system elements and a way to reorganize the structure to respond to external change

- Ability to organize connections into larger components with consequent changes to connections and interactivity

- Aggregation of smaller units into larger components 
All of these become check points in a design. Not each of these is relevant to each level of design.

\subsection{The Impact on Systems Modeling}

Writers such as Merali [9, 10] or Kovacs [11] suggest that IS system design no longer focus on the design of deterministic systems that attempt to reduce complexity through structure but on systems that support evolution and change.

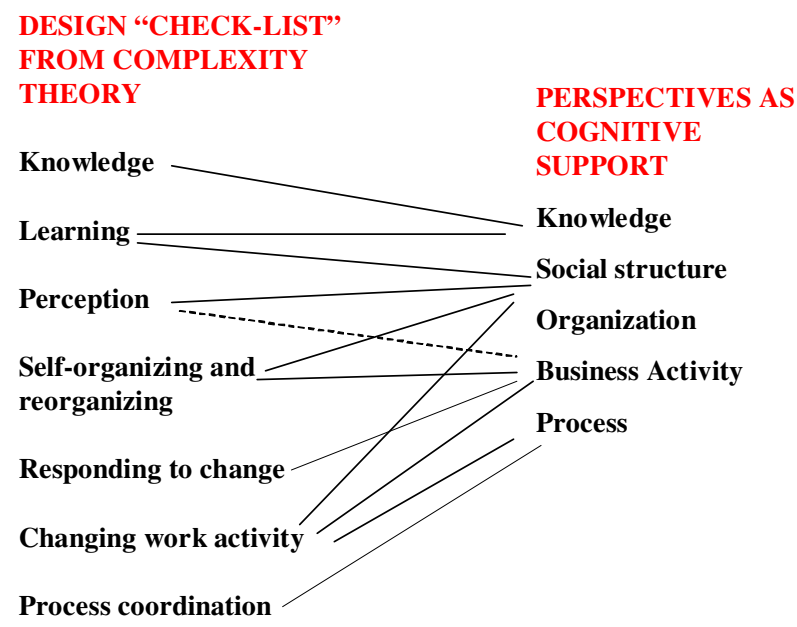

Fig. 2. Design Checklist

The complex business environment requires process management that goes beyond managing simple workflows but require systematic ways to manage complexity. These require systems to support the design criteria described in the previous section. The paper proposes that such design criteria can be met from the following perspectives:

- The business activities and their actions and what they create,

- The process workflow or sequence of activities and the interdependence between activities,

- The social structure that describes roles and their responsibilities and the assignment of roles to individuals to describe the increasing importance of social interactions in any design.

- The knowledge created and used during the activities, and

- The technology and how it can be used to assist process management.

Figure 2 shows the relationship between the criteria and perspectives. For example learning is related to the knowledge and social perspectives as learning requires the sharing and creation of knowledge within social environments. 


\section{Choosing Modeling Methods}

The options for designers of systems that satisfy complex criteria are:

- Using the traditional methodologies to model other perspectives,

- Extending existing methodologies with new perspectives either by providing new modelling techniques or extending current modelling structures, or

- $\quad$ Creating new modelling methods.

Traditional methods include various project management tools, or modelling methods such as E-R or workflow modelling, which have been successful in developing structured systems in the past. Their purpose is to define the terms needed to describe systems in terms natural to users and then a way to convert models in these terms to computer systems. There are now a number of such models in practice mainly used to develop structured deterministic systems. These do not contain specific constructs to address the new criteria introduced through complexity. The alternative described here is to develop models for each of the perspectives and to integrate the models into a holistic system.

\subsection{Choosing the Semantics}

The paper describes the kinds of concepts used to model the different perspectives and ways to integrate them [12]. It focuses on using the knowledge perspective as central driver in the more emergent knowledge based processes.

Figure 3 illustrates a modeling method called the business activity model (BAM) that shows the combination at a high level. It includes concepts both from the business, social and knowledge perspectives. It is principally a high level diagram that shows the main entities in the system. It uses concepts of a conceptual model for collaborative systems [13]. These focus on collaborative business systems and have been verified in earlier research $[14,15]$. As shown in Figure 3, the main modelling concepts are the activity (shown as ellipses), role (shown as black dots), and artefact

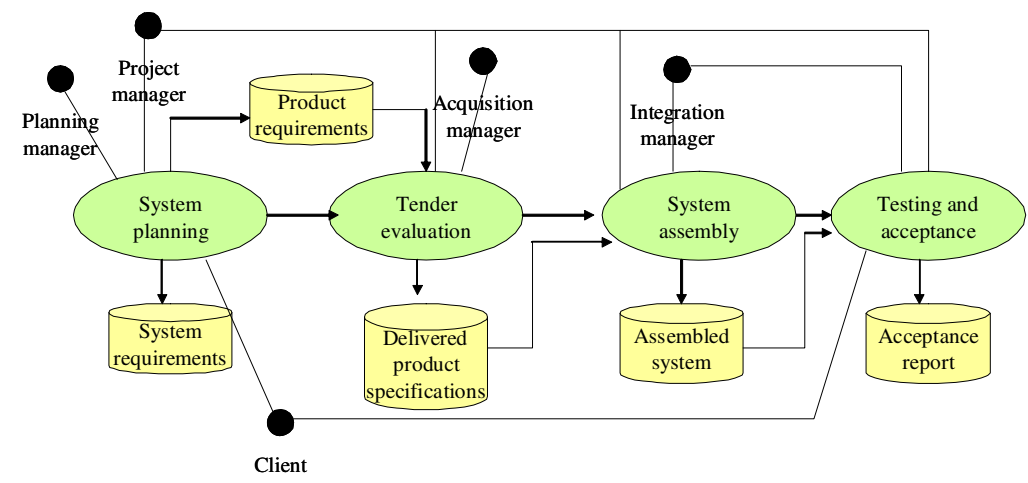

Fig. 3. Process for system development 
(shown as disk shapes). It is also possible to add participants or people who tale on the roles by attaching their names to the roles. Figure 3 illustrates one instance of such model showing the main activities in a tendering process to create a new engineering system. The Figure also illustrates the links to both social structures through roles and to explicit knowledge through artifacts. Later knowledge creation through interactions is described using the enterprise social network (ESN).

Figure 3 shows the following activities:

- System planning where client requirements are developed. These specify the various components needed to construct the system,

- Tender construction for the components and evaluation of response,

- System assembly of delivered products, and

- Testing and Acceptance of the constructed system.

The activities in Figure 3 are on-going. The ability to self organize is through the governance structure within the activity. Learning and knowledge are specified as responsibilities with the social structure, which is modelled by the ESN illustrated in Figure 4.

The ESN diagram is introduced in this paper as an extension of social networks. The roles here define responsibilities of people assigned to the roles. It includes the following concepts:

- Roles that define responsibilities. These responsibilities are shown by the attached text; for example, the project manager organizes the project. One important responsibility defined at this level focuses on knowledge and learning. Thus for example the project manager needs to develop knowledge on improving project management techniques in their environment,

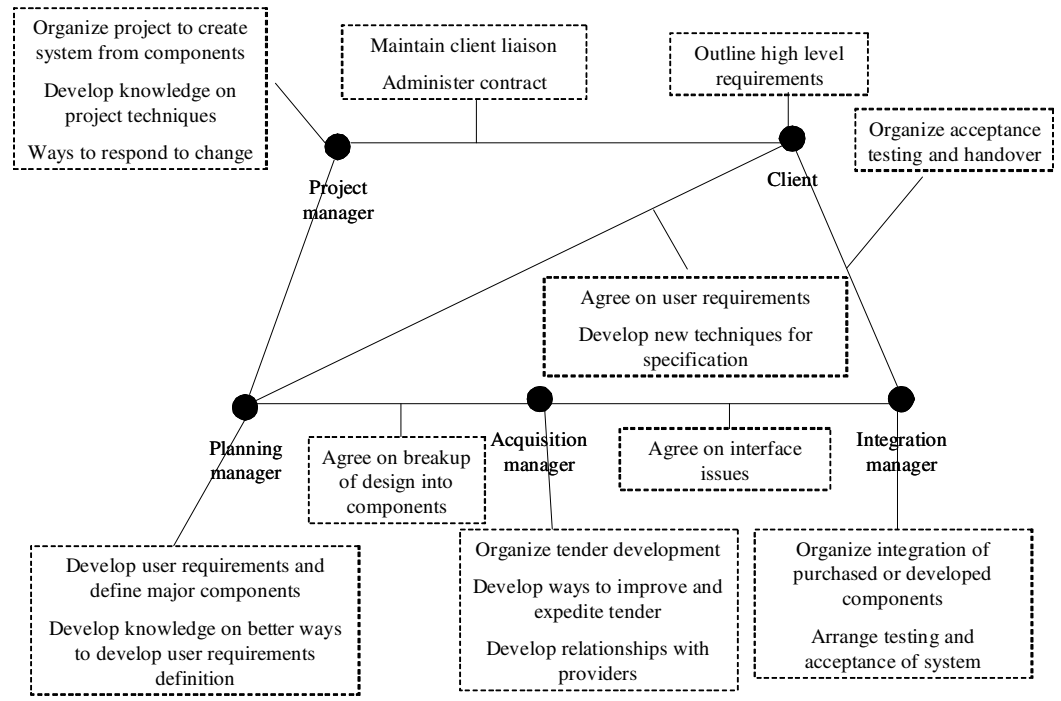

Fig. 4. ESN Diagram 
- Participants who take on these roles can be shown by faces;

- Interactions shown by lines between the roles showing the kind of interactions between people assigned to these roles; for example the major interaction between client and integration manager is to organize acceptance tests.

As is normal in most design processes the high level business activities are described at lower levels. Figure 5 for example illustrates the expansion of system planning into more detailed business activities

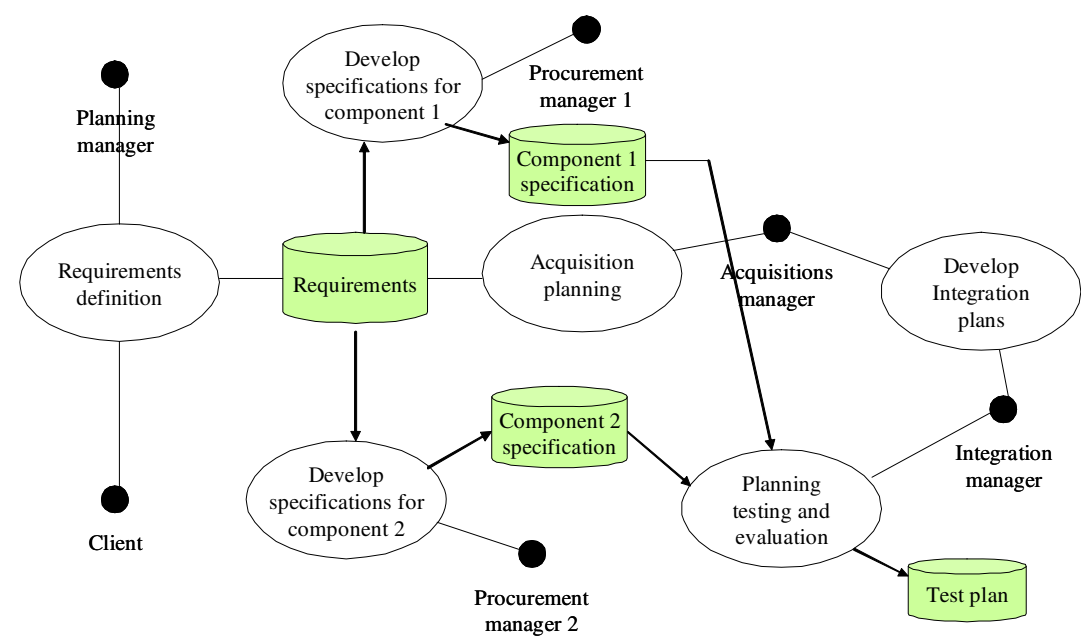

Fig. 5. System planning activities

The activities are now shown in more detail that includes:

- The definition of requirements

- The decomposition of the requirements into components and identifying the component specifications to tendering teams,

- Specifying acquisitions planning to ensure components are delivered as needed,

- Development of integration plans to put the components together, and

- $\quad$ Specifying the test procedures

These specifications are used in later stages.

Knowledge is gathered during the system planning stage to be used later in the tendering and system construction activities as well as in integration and testing.

\subsection{Integrating the Knowledge and Social Perspectives in the Business Context}

The enterprise social network (ESN) is also constructed for lower level business activities. It shows the responsibilities of the project manager in more detail and introduces any additional roles found at lower level activities. In this case these procurement managers who will be later responsible for developing tenders and 
accepting supplied components. Their main responsibility during planning is to develop the component specifications that are later used to construct the tender. The ESN contains an additional construct to indicate interactions between three or more roles. Thus for example the procurement managers and planning manger together interact to develop the acquisitions schedule and tender requirements.

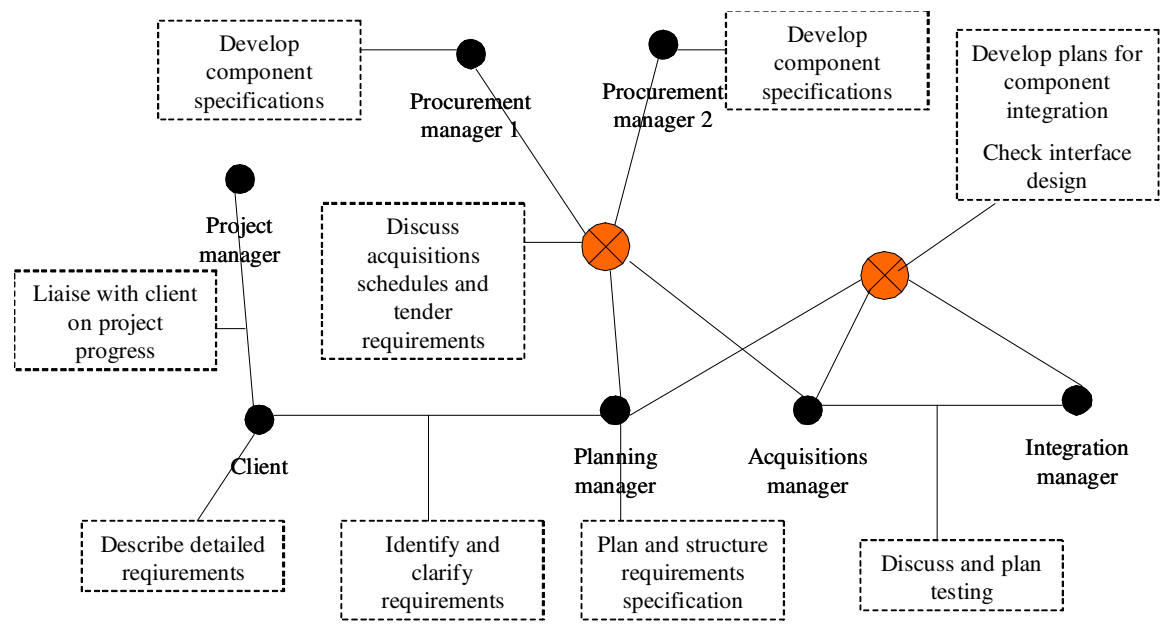

Fig. 6. Enterprise Social Network for System Planning

Figure 5 describes the social network in the system. Here each role is represented by a black circle.

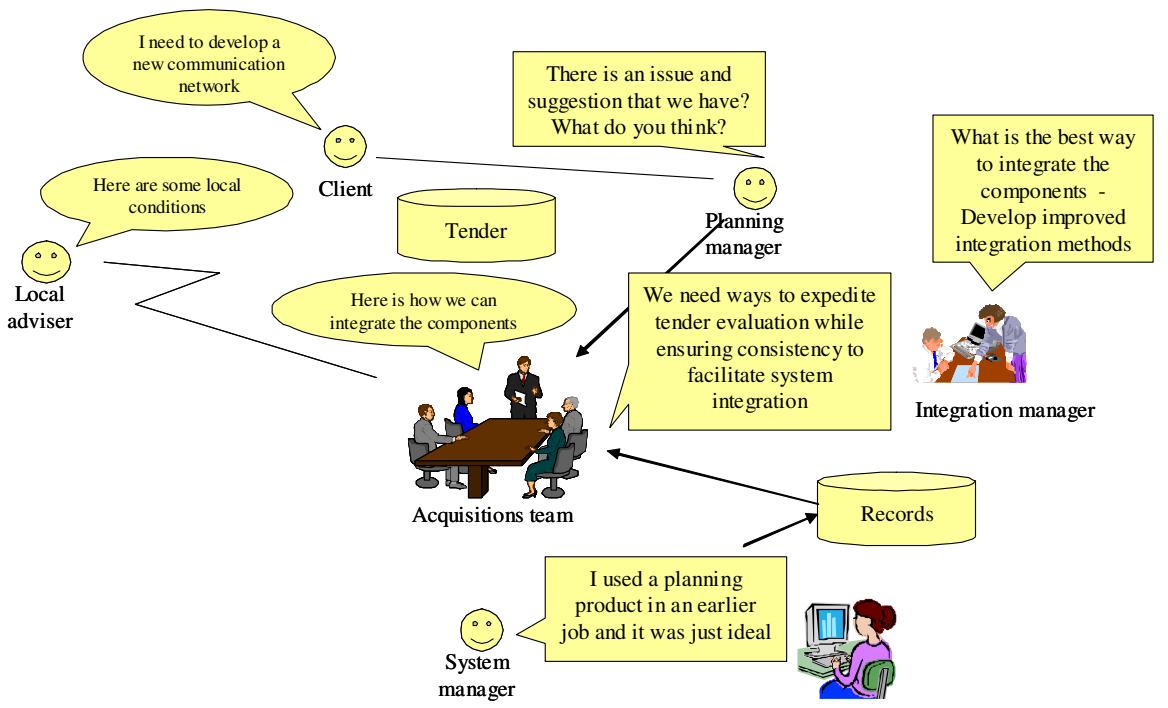

Fig. 7. A Rich Picture Model of the Knowledge Perspective 


\subsection{Modeling the Knowledge Perspective}

Knowledge is a less structured and visible perspective and it can be shown using rich pictures that illustrate the knowledge needs of the different roles. The goal here is to capture the knowledge needed to improve activities.

The paper now shows the integration of the knowledge and social perspectives.

\subsection{Specifying Change in Terms of Perspective Semantics}

Change can now be specified in different perspectives and easily converted to implementation. The changes specified in Table 1 can be described in terms of the semantic concepts. For example:

Change to the organization can be implemented by creating new business activities, then adding roles and interactions as needed,

Changes to the activity can be described by changing its roles or artifacts,

Changes to the role can be expressed by changes to role responsibilities and interactions.

Assign a person to a role is expressed by linking a participant to a role.

The next requirement is for such models and their creation and change to be directly implemented using software.

\section{Defining the Supporting Technology Infrastructure}

The two steps to be satisfied in an implementation are to identify the services needed to support the interactions within the system and ways to integrate the services into platforms that present a holistic environment to system participants.

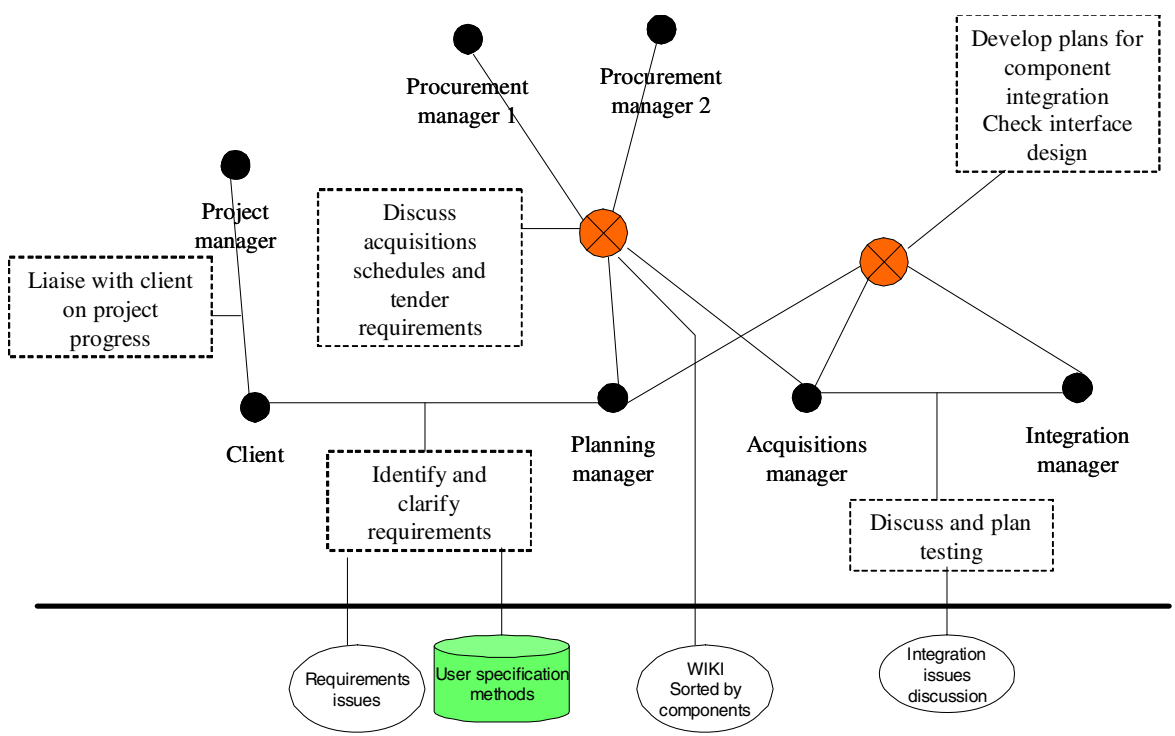

Fig. 8. Collaborative Infrastructure 


\subsection{Identifying the Required Services}

Services must be chosen to support the interactions between the roles in the system. The main aspect of collaboration is to support the interactions between the different roles. The choice is illustrated in Figure 8. The interactions in the ESN are now mapped to social software. For example a blog is provided for client discussions, whereas alliance discussions are supported by a WIKI.

\subsection{Software Infrastructure Requirements}

Most current systems are supported by workflow technologies that follow a predefined set of steps. Any required social networking is carried out outside such systems using a limited set of collaborative technologies. Knowledge sharing between the two is often minimal. Complex dynamic systems that align the collaborative interactions to formal processes are better supported by:

- Middleware - this provides a solution where workspaces can be customized to roles with links to corporate databases. They can be used to develop special interfaces for roles or activities. However middleware change is more difficult than change using lightweight technologies, and the expectation is that change would not happen frequently. In most cases it would require information technology specialists to construct an interface for each individual and change it as needed.

- Lightweight technologies - these provide better abilities for change but in many cases cannot easily connect to corporate wide databases or other lightweight systems. They can be used to develop the one-fits-one option or for mass personalization, which is ideal for knowledge workers. Many allow users themselves can create and manage their workspace.

Software must be chosen to support change specified in terms of natural semantics. Thus software must include commands that actually create a workspace, add a new role, setup a new interaction and place it in the context of the activity. Lightweight platforms are an important option. However to support user driven change they must provide users with commands based on the modeling concepts as a guideline. They should include the concepts defined for the collaborative model while providing commands to easily create and change the structures of workspaces. Our experimental system, LiveNet, demonstrates the kind of support needed by workspace systems. Figure 9 shows the LiveNet interface and its typical commands.

It provides a menu that can be used to create new collaborative objects, including activities, roles, and artifacts. It also enables people to be assigned to the roles. Apart from these elementary operations the system includes ways to implement governance features as for example allowing roles limited abilities to documents. The system includes support for sharing artifacts across workspaces and a permissions structure to control such sharing. Social software such as blogs or discussion systems is supported and can be shared across workspaces. 
Commercial systems in this area focus on middleware software that provides the commands that allows users to use the middleware functionality to create workspaces. Furthermore, it should allow users to change the workspaces as work practices change. Many manufacturers are now providing ways to integrate the kind of software with enterprise applications. A typical example here is Websphere provided by IBM. The challenge in many such systems is to provide ways to share knowledge across activities. They provide access to corporate databases but often do not support the sharing of knowledge collected in the course of knowledge work in identifying and solving problems, and making decisions.

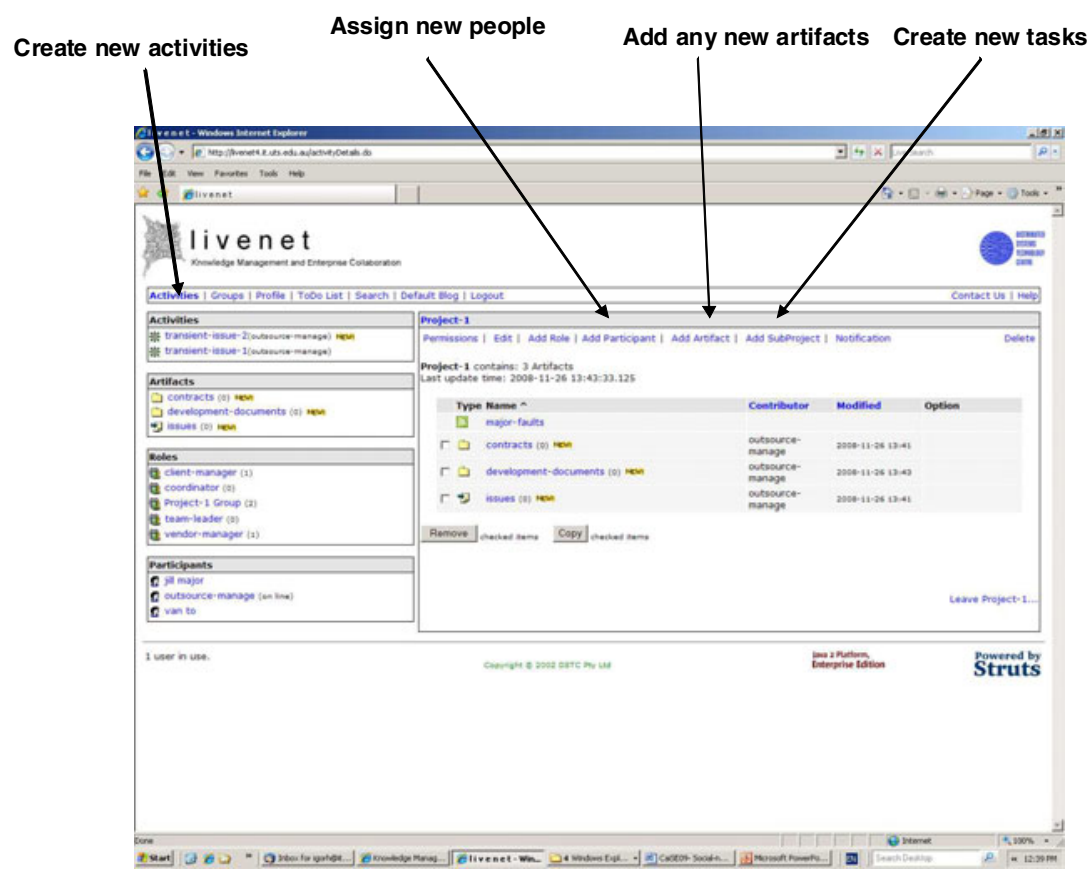

Fig. 9. A Demonstration Workspace

\section{Summary}

The paper began by describing the increasing complexity of business processes and a systematic way to describe it. It described how complexity adds new criteria to design processes and discussed the implication for system modelling. It suggested that such criteria can be met by seeing systems from a number of perspectives and using the perspectives to specify ways to meet the criteria. It developed models to represent the perspectives and illustrated them in the context of tendering processes. 


\section{References}

1. Heikkila, J.: From supply to demand chain management: efficiency and customer satisfaction. Journal of Operations Management 20, 747-767 (2002)

2. Howard, M., Vidgen, R., Powell, P.: Automotive e-hubs: Exploring Motivations and barriers to collaboration and interaction. Journal of Strategic Information Systems 15, 51-75 (2006)

3. Rye, K., Lee, S., Choi, H.: Modularization of web-based collaboration systems for manufacturing Innovation. In: Proceedings of the Tenth International Conference on Enterprise Information Systems, Barcelona, June 12-16, pp. 174-177 (2008)

4. Prahalad, C.K., Krishnan, M.S.: The New Age of Innovation. McGraw-Hill, New York (2008)

5. Vidgen, R., Wang, X.: From business process management to business process ecosystem. Journal of Information Technology 21, 262-271 (2006)

6. Cova, B., Salle, R.: Marketing solutions in accordance with S-D logic: Co-creating value with customer network actors. In: Industrial Marketing Management, vol. 37, pp. 270-277. Elsevier Press, Amsterdam (2008)

7. McElroy, M.W.: Integrating Complexity theory, knowledge management and organizational learning. Journal of Knowledge Management 4(3), 195-203 (2000)

8. Hawryszkiewycz, I.T.: Supporting Complex Adaptive Systems with Lightweight Platforms. In: Luis, M., Camerinha-Matos, P.W. (eds.) Pervasive Collaborative Networks: IFIP TC 5 Ninth Working Conference on Virtual Enterprises, Poznan, Poland, September 2008, pp. 381-388. Springer, Heidelberg (2008)

9. Merali, Y., McKelvey, B.: Using Complexity Science to effect a paradigm shift in Information systems for the 21st century. Journal of Information Technology 21, 211-215 (2006)

10. Merali, J.: Complexity and Information Systems: the emergent domain. Journal of Information Technology 21(4), 216-233 (2006)

11. Kovacs, A.I., Ueno, H.: Towards Complex Adaptive Information Systems. In: Proceedings of the 2nd International Conference on Information Technology and Application (2004)

12. Hawryszkiewycz, I.T.: Knowledge Management: Organizing the Knowledge Based Enterprise. Palgrave-Macmillan, Basingstoke (2010)

13. Hawryszkiewycz, I.T.: A Metamodel for Modeling Collaborative Systems. Journal of Computer Information Systems XLV(3), 63-72 (Spring 2005)

14. Hawryszkiewycz, I.T.: Providing Computer Services For Business Networks. In: Proceedings of the Ninth International Conference on EDI-IOS, Bled, pp. 398-411 (June 1996), ISBN-961-232-000-4

15. Hawryszkiewycz, I.T.: A Framework for Strategic Planning for Communications Support. In: Proceedings of The Inaugural Conference of Informatics in Multinational Enterprises, Washington, pp. 141-151 (October 1997) 